\title{
EVALUACIÓN DEL COMPORTAMIENTO DE VIGAS DE CONCRETO REFORZADAS Y SUBREFORZADAS CON VARILLAS DE PRFV
}

EVALUATION OF THE BEHAVIOR OF REINFORCED CONCRETE BEAMS AND UNDER-FORCED WITH GFRP RODS

\author{
Juan Alberto Sánchez Hernández ${ }^{1}$ \\ juan-sanchez@proyesting.com \\ Jorge Alfredo Aguilar Carboney ${ }^{2}$ \\ jaguilar@unach.mx \\ Manuel Jara Díaz ${ }^{3}$ \\ mjarad10@gmail.com
}

1 Comisión de Estructuras del Colegio de Ingenieros Civiles de Chiapas, MÉXico

2 Facultad de Ingeniería. Universidad Autónoma de Chiapas, México

3 Facultad de Ingeniería. Universidad Michoacana de San Nicolás de Hidalgo, México 
Para citar este artículo:

Sánchez Hernández, J. A., Aguilar Carboney, J. A., \& Jara Díaz, M. (2021). Evaluación del comportamiento de vigas de concreto reforzadas y subreforzadas con varillas de PRFV. ESPACIO I+D, INNOVACIÓN MÁS DESARROLLO, 10(26). https://doi.org/10.31644/ IMASD.26.2021.a04

\section{RESUMEN}

Esta investigación evalúa el comportamiento y el diseño de vigas simplemente soportadas, hechas de concreto armado con barras de polímero reforzado con fibra de vidrio (GFRP-glass fiber reinforced polymer) sometidas a una carga simétrica. El propósito del trabajo es verificar las expresiones y consideraciones del código de diseño ACI 440 1R (2015) en las vigas de confinamiento (dalas) que generalmente se usan para huecos de puertas y ventanas en edificios residenciales. Para ello, se prueban experimentalmente en el laboratorio dos vigas de concreto armado con GFRP y cuantía mínima de acuerdo con el ACI 440 1R, de sección $15 \times 20 \mathrm{~cm}$ para determinar su capacidad y comportamiento mecánico. Como resultado, se encuentra que la resistencia obtenida hasta el fallo, tanto en corte como en flexión, fue mayor de lo esperado según la metodología de análisis. Los resultados de estas pruebas complementan el trabajo realizado por Sanchez Hernández (2019b).

\section{Palabras Clave}

Polímeros reforzados; fibras de vidrio; diseño subreforzado; concreto reforzado. 
This work evaluates the behavior and design of simply supported beams, made of reinforced concrete with glass fiber reinforced polymer (GFRP) bars subjected to a symmetrical load. The purpose of the work is to verify the expressions and considerations of the ACI 440 1R (2015) design code on the confinement beams (dalas) that are generally used in the perimeter of door and window in residential buildings. For this, two reinforced concrete beams with GFRP and a minimum quantity according to ACI 440 $1 \mathrm{R}$, with a section $15 \times 20 \mathrm{~cm}$, are tested in the laboratory to determine their capacity and mechanical behavior. As a result, it is found that the resistance obtained until failure, both in shear and in bending, was greater than expected according to the analysis methodology. The results of these tests complement the work carried out by Sanchez Hernández (2019b).

\section{Keywords}

Reinforced polymers; glass fibers; under-reinforced design; reinforced concrete. 
$\mathrm{E}$ 1 uso en la construcción de los compuestos poliméricos reforzados con fibra (FRP- Fiber Reinforced Polimer) ha tomado gran auge en las últimas dos décadas, debido al costo competitivo que tuvo la inclusión de la fibra de vidrio como refuerzo del compuesto y a sus ventajas de durabilidad, alta resistencia a la tensión, bajo peso y ausencia de corrosión en los ambientes donde el acero falla (Sánchez Hernández J. A., 2019a). No obstante, en Latinoamérica su aceptación es aún limitada debido a que es un material novedoso que despierta el interés de la industria de la construcción por conocer el comportamiento de este tipo de refuerzos en elementos estructurales comúnmente empleados en edificaciones habitacionales típicas

El polímero reforzado con fibra de vidrio (FRPV) a diferencia del acero de refuerzo, presenta una curva elástica hasta la falla cuando es sometida a tensión además de ser anisótropo, es decir, presenta diferencias entre su resistencia a tensión, corte y compresión. Como ya se mencionó, el material carece de un límite de fluencia que compensa con su alta resistencia y un módulo elástico bajo, lo cual le confiere capacidad de deformación y respuesta dúctil al binomio concreto-refuerzo. Sin embargo, esto genera incertidumbre sobre la seguridad del diseño (GangaRao, Taly, \& Vijay, 2007) enfocado en la ductilidad del acero. Resultando importante por ello verificar que la metodología de diseño expuesto por el ACI $4401 \mathrm{R}$ brinde una seguridad aceptable ante las demandas analizadas.

Las pruebas se realizaron en dos etapas, debido a la disponibilidad del material, siendo el primer conjunto de ellas enfocadas en garantizar la seguridad de los usuarios de este producto de recién ingreso al mercado y su funcionamiento a flexión, en tanto que en la segunda etapa que se refleja en este informe fue el de comprobar la respuesta al corte. Los ensayos previos mostraron un comportamiento conservador de los elementos ante el diseño a flexión con una cuantía de refuerzo muy por debajo del mínimo normativo (Sánchez Hernández J. A., 2019b), en este experimento se evalúa una cuantía de refuerzo a flexión apegada a los mínimos que suelen usarse en la autoconstrucción con elementos electrosoldados de acero y permitidos por las Normas Técnicas Complementarias del Reglamento de Construcciones vigentes en la Ciudad de México (NTC M, 2017) para refuerzos en elementos de confinamiento horizontal y particularmente en dalas de cerramientos de puertas y ventanas típicas.

Los resultados analizan y presentan en sistema MKs para facilitar la interpretación del constructor promedio.

\section{DESCRIPCIÓN DE LA PRUEBA}

Caracterización del espécimen. Se ensayaron dos vigas de concreto de sección transversal rectangular de $15 \times 20 \mathrm{~cm}$ armadas mediante varillas de PRFV de acuerdo con lo indicado en la Tabla 1, con estribos de $4 \mathrm{~mm}$ diámetro a cada 
$15 \mathrm{~cm}$ de espaciamiento. La viga V3 se armó en el lecho superior con dos varillas de diámetro $4 \mathrm{~mm}(2 \mathrm{D} 4)$ y en el lecho inferior con dos varillas de 4 mm de diámetro más una varilla de $6 \mathrm{~mm}$ de diámetro (1D6), con ganchos (G) de $4 \mathrm{~mm}$ y $6 \mathrm{~mm}$ en sus extremos inferiores. En tanto que la viga $\mathrm{V}_{4}$ su armado fue con dos varillas de $6 \mathrm{~mm}$ en el lecho superior y 3 varillas de $8 \mathrm{~mm}$ en el inferior, adicionadas con sus respectivos ganchos. El área de refuerzo en el lecho inferior se denominó Af y se resume también en la citada Tabla. Así mismo se muestra la resistencia (f'c) probada a 28 días de los concretos empleados en las vigas y las cuantías balanceada, mínima y real obtenidas para los especímenes de las pruebas.

\section{Tabla 1}

Descripción de Especímenes

\begin{tabular}{|c|c|c|c|c|c|c|}
\hline \multirow[b]{2}{*}{ VIGA } & \multirow[b]{2}{*}{ ARMADO } & \multirow[b]{2}{*}{$\mathrm{f}^{\prime} \mathrm{c} \mathrm{kg} / \mathrm{cm}^{2}$} & \multirow[b]{2}{*}{ Af $\mathrm{cm}^{2}$} & \multicolumn{3}{|c|}{ CUANTÍA } \\
\hline & & & & $\rho_{\mathrm{fb}}$ & $\rho_{\text {fmin }}$ & $\rho_{\text {fREAL }}$ \\
\hline $4(v 3)$ & $\begin{array}{c}\text { LS 2D4 } \\
\text { LI 2D4+1D6+G } \\
\text { E@15 }\end{array}$ & 279.80 & 0.53 & 0.0060 & 0.0031 & 0.0020 \\
\hline $5(\mathrm{v} 4)$ & $\begin{array}{c}\text { LS 2D6 } \\
\text { LI 3D8+G } \\
\text { E@10 }\end{array}$ & 218.50 & 1.51 & 0.0047 & 0.0031 & 0.0057 \\
\hline
\end{tabular}

Las pruebas de cilindros de concreto a los 28 días arrojaron resultados de f'c entre $200 \mathrm{~kg} / \mathrm{cm}^{2}$ y $280 \mathrm{~kg} / \mathrm{cm}^{2}$, etiquetándose como V3 y V4 respectivamente. El valor del esfuerzo a tensión de la barra de refuerzo se fijó inicialmente con $\mathrm{ff}^{*} \mathrm{u}=8000 \mathrm{~kg} / \mathrm{cm}^{2}$ (esfuerzo límite último de tensión establecido por el fabricante), $\mathrm{ffv}=1500 \mathrm{~kg} / \mathrm{cm}^{2}$ (esfuerzo límite de corte establecido por el fabricante), $\mathrm{Ef}=500000 \mathrm{~kg} / \mathrm{cm}^{2}$ (módulo elástico establecido por el fabricante). Dichas varillas de PRFV presentaron en ensayes realizadas en el Instituto Mexicano de Cemento y el Concreto, A.C. y el Organismo Nacional de Normalización y Certificación de la Construcción y Edificación, S.C. (ONNCCE, 2016) resistencias promedio de hasta $\mathrm{ffu}=13700 \mathrm{~kg} / \mathrm{cm}^{2}$ y módulo elástico de $\mathrm{E}=669000 \mathrm{~kg} / \mathrm{cm}^{2}$. Es decir, un margen de capacidad por encima del indicado por el proveedor.

De acuerdo con las ecuaciones (7.2.4) y (6.2 a) del ACI 440 1R (2015), se establece el refuerzo longitudinal mínimo requerido:

$$
\begin{gathered}
\mathrm{Af}=1.31 \mathrm{~b} \mathrm{~d}\left(\mathrm{f}^{\prime} \mathrm{C}^{1 / 2}\right) / \mathrm{ffd} \geq 23.43 \mathrm{~b} \mathrm{~d} / \mathrm{ffu} \quad(\text { ACI } 440.1 \mathrm{R} \mathrm{7.2.4}) \\
\mathrm{ffd}=\mathrm{CE} \mathrm{ffu} \quad(\text { ACI } 440.1 \mathrm{R} 6.2 \mathrm{a})
\end{gathered}
$$


Donde:

$$
\mathrm{ffu}=8,000 \mathrm{~kg} / \mathrm{cm}^{2} \mathrm{y} \mathrm{ffd}=\mathrm{CE} \mathrm{ffu}=6,500 \mathrm{~kg} / \mathrm{cm}^{2}
$$

Por lo que considerando $\mathrm{r}=2.5 \mathrm{~cm}, \mathrm{~d}=20-2.5=17.5 \mathrm{~cm}$ y f' $\mathrm{c}=250 \mathrm{~kg} / \mathrm{cm}^{2}$, se tiene:

$$
\begin{gathered}
\text { Af }=1.31(15 \mathrm{~cm})(17.5 \mathrm{~cm})\left(250 \mathrm{~kg} / \mathrm{cm}^{2}\right)^{1 / 2} /\left(6,500 \mathrm{~kg} / \mathrm{cm}^{2}\right) \geq 23.43(15 \mathrm{~cm}) \\
(17.5 \mathrm{~cm}) /\left(8000 \mathrm{~kg} / \mathrm{cm}^{2}\right) \\
\text { Af }=0.84 \mathrm{~cm}^{2} \geq 0.77 \mathrm{~cm}^{2}=0.84 \mathrm{~cm}^{2}
\end{gathered}
$$

Por esta razón, en la prueba se consideraron dos cuantías de refuerzo, una ligeramente por debajo y otra ligeramente por encima del refuerzo mínimo evaluado por la ecuación 7.2.4 del ACI 440 1R (2015).

Descripción de los ensayos. Los ensayos se realizaron en un marco de reacción como el descrito en la Figura 1, el cual emplea un gato hidráulico de cien toneladas para transmitir la carga simétrica en el elemento a través de una viga de distribución de carga lo suficientemente robusta para no deformarse. La viga fue colocada en los apoyos isostáticos incluidos en la parte inferior del marco constituidos por un apoyo simple y un apoyo articulado. De esta forma el experimento pretende eliminar la incertidumbre de las condiciones de apoyo, simplificando las condiciones del ensaye para apegarse al modelo matemático usado en el análisis numérico.

Procedimiento de carga. Las pruebas se realizaron mediante la aplicación de carga a una velocidad constante de $40 \mathrm{~kg} / \mathrm{s}$, lo que permite la observación del comportamiento del elemento, en cuanto a sus deflexiones y agrietamiento a través del tiempo.

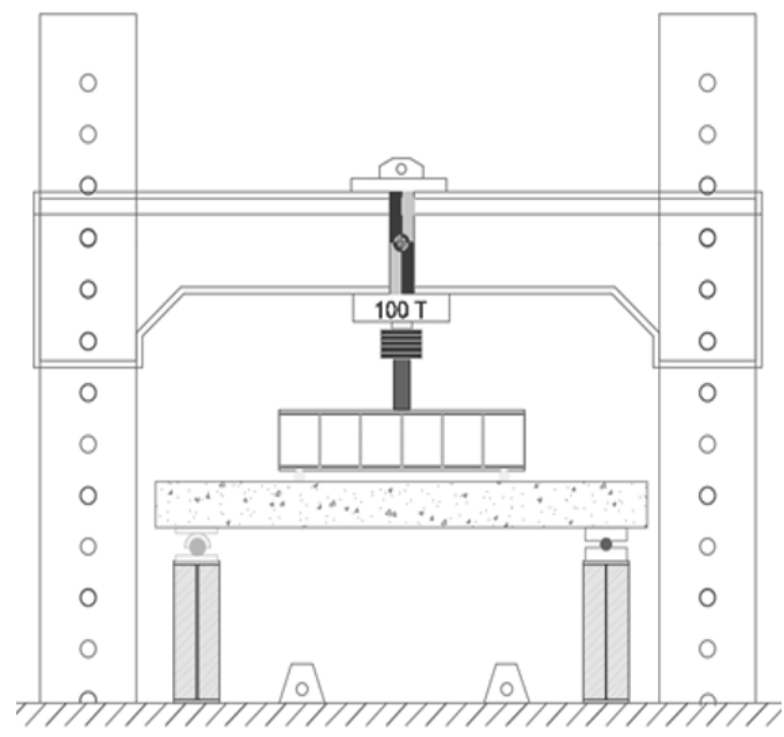

Figura 1. Esquema del arreglo para el ensaye a flexión 
Descripción del análisis. En el cálculo de la resistencia a flexión de diseño se empleó el modelo de distribución de esfuerzos y deformaciones establecido en las NTC Ciudad de México (2017); para vigas de concreto reforzados con varillas de acero y que describen Hidalgo Toxqui et al (2018) apegados al ACI 440 1R (2015) en el análisis de vigas reforzadas con varillas de PRFV según la Figura 2 y los cálculos que se siguen respecto a la flexión.

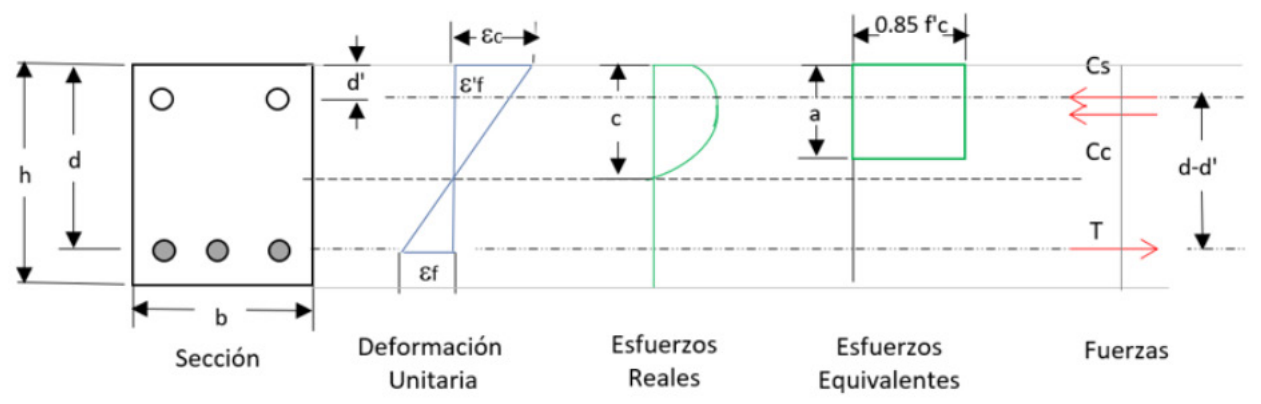

Figura 2. Diagramas de distribución de esfuerzos y deformaciones en vigas

En el modelo descrito por la Figura 2, donde se representa el armado principal a flexión simple con círculos grises y el secundario por círculos blancos, el momento nominal está dado por la expresión:

$$
\mathrm{Mn}=\operatorname{Af}(\mathrm{ffd})(\mathrm{d}-\beta 1 \mathrm{c} / 2) \quad(\text { ACI } 4401 \mathrm{R} \text { 7.2.2e }) \quad \text { (Ecuación 1) }
$$

Af se refiere al área de refuerzo de la sección transversal de las varillas, de acuerdo a la Tabla 1; ffd al esfuerzo límite de diseño, evaluado en la ecuación 6.2 (a) del ACI 440 1R (2015), arriba indicado; $\rho f b$ o cuantía balanceada se evalúa de acuerdo con el ACI 440 1R 7.2.1(b), con $\beta 1=0.85$ :

$$
\rho f b=0.85 \beta 1\left(f^{\prime} c / f f d\right)[0.003 \mathrm{Ef} /(0.003 \mathrm{Ef}+\mathrm{ffd})] \text {, donde } \rho f b>\rho f
$$

Para evaluar la resistencia a la fuerza cortante, debido al bajo módulo elástico del PRFV, comparado con el acero, se suponen agrietamientos más profundos, y por consecuencia una disminución sustancial de la resistencia transversal de la viga. Por ello se evalúa la contribución del concreto $(V c R)$ en función de la profundidad del eje neutro de la sección agrietada, expresado de la siguiente manera:

$$
V c R=F R 1.28 \sqrt{f^{\prime} c} \mathrm{~b}(\mathrm{kd}) \quad(\text { ACI } 4401 \mathrm{R} 8.2 \mathrm{a}) \quad(\text { Ecuación 2) }
$$


Donde:

$k=\sqrt{\left[2(\rho f) n f+((\rho f) n f)^{2}\right]}-(\rho f) n f$ de acuerdo al (ACI 440 1R 7.3.2.2b) y $n f=\mathrm{Ef} / \mathrm{Ec}$.

En lugar del modelo indicado por las NTC CR (2017):

$$
V c R=\operatorname{FR}(0.2+20 \mathrm{r}) \sqrt{f^{\prime} c} \text { bd } \quad \text { bd } \quad(\text { NTC CR 5.3.1) (Ecuación 2.2) }
$$

En tanto que la contribución del refuerzo (VfR) se tomará en cuenta considerando el mismo modelo analítico descrito en las NTC CR (2017), inciso (5.3.29) y ACI 440 1R:

$$
V f R=(\text { FR Afv ffv d }(\operatorname{sen} \theta \cos \theta) / s \quad(\text { ACI } 440 \text { 1.R 8.2f })
$$

Donde: $\theta$ = ángulo de inclinación de los estribos y Afv = área de estribos a corte.

$$
\text { Siendo la Resistencia total: } V R=V f R+V c R \quad \text { (Ecuación 2.3） }
$$

La Figura 3 muestra el modelo analítico para la determinación del momento y la cortante actuante para los ensayos del experimento, así como las condiciones geométricas de la aplicación de la carga simétrica P durante el ensayo.
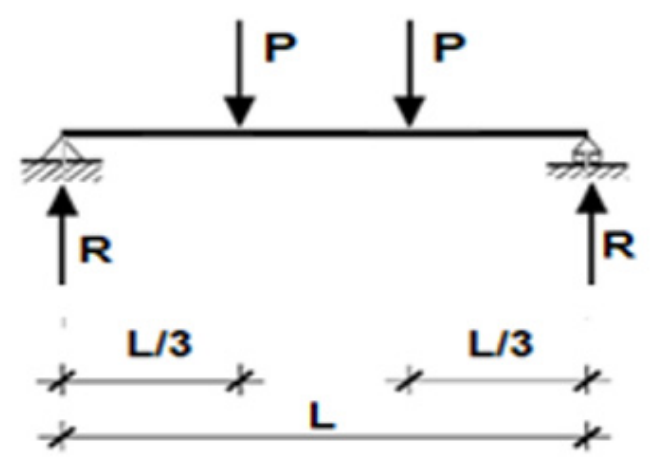

Figura 3: Gráfico del modelo analítico para el cálculo de los elementos mecánicos

El momento y cortante actuante máximo se obtienen de las siguientes ecuaciones:

$$
\begin{array}{ll}
\mathrm{M}=\mathrm{P}(\mathrm{L} / 3) & (\text { Ecuación 3) } \\
\mathrm{V}=\mathrm{P} & (\text { Ecuación 4) }
\end{array}
$$


Donde:

$$
\mathrm{M}=\text { Momento actuante; } \mathrm{P}=\text { Carga; } \mathrm{L}=\text { Claro entre apoyos. }
$$

El comportamiento a flexión se evaluará mediante la comparación de las ecuaciones que determinan tanto el momento actuante (Ecuación 3) de cada ensayo durante la aplicación progresiva de la carga con el momento teórico máximo determinado analíticamente por (Ecuación 1). En tanto que la resistencia a la fuerza cortante se medirá mediante la comparación de la capacidad máxima analítica determinada conforme a la ecuación 2.3 y el desarrollo de la resistencia del ensayo de acuerdo con la Ecuación 4.

\section{Comparación y análisis de resultados}

La Figura 4 muestra las gráficas de carga $2 \mathrm{P}$ obtenida durante el experimento en función de las deflexiones centrales $\Delta$. En ella se observan variaciones de la carga resistente, que puede ser explicada como corrimientos del refuerzo debido al acomodo por adherencia entre la varilla de PRFV y el concreto; no obstante, existe una redistribución de esfuerzos que le permiten al elemento continuar trabajando e incrementar su resistencia hasta la falla definitiva. También se puede observar que a mayor cuantía de refuerzo ( $\rho$ de $\mathrm{V}_{4}>\rho$ de $\mathrm{V}_{3}$ ), existe mayor uniformidad en el comportamiento de la curva.

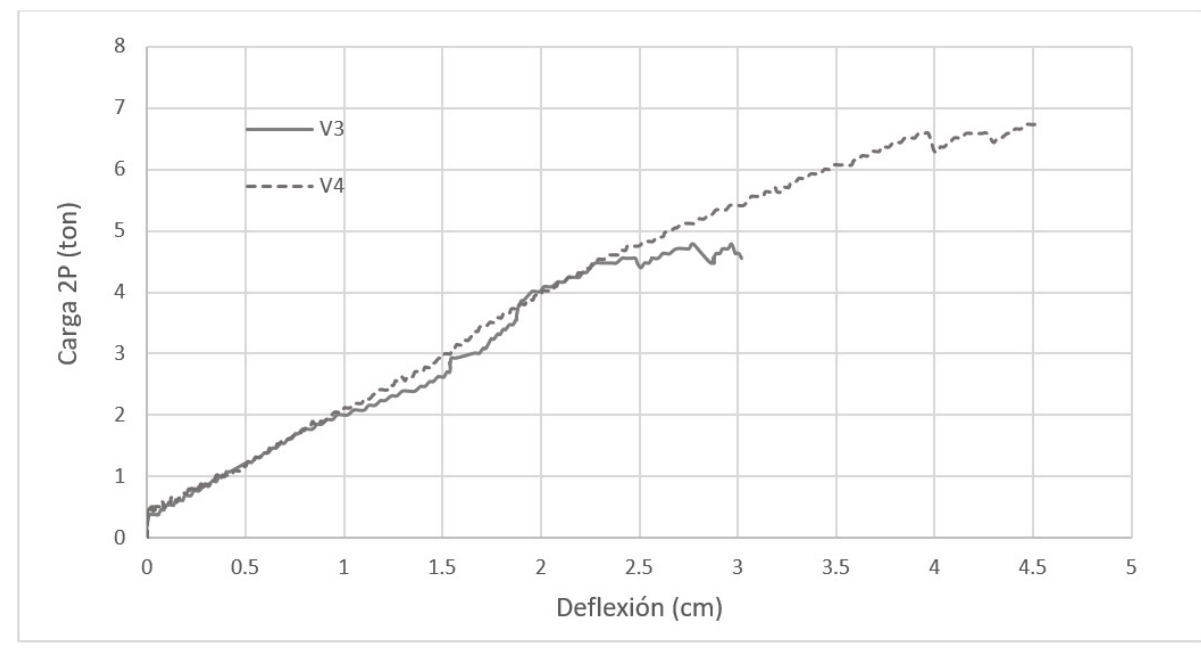

Figura 4. Gráfica de Carga 2P x $\Delta$

Al evaluar el modelo utilizado en las hipótesis para la obtención de resistencias de diseño a flexión de acuerdo con las ecuaciones 1 y 2.3, se obtuvieron esfuerzos de registro mayores a $\mathrm{ff} * \mathrm{u}=8,000 \mathrm{~kg} / \mathrm{cm}^{2}$ y ffv $=1,500$ $\mathrm{kg} / \mathrm{cm}^{2}$ nominales garantizados por el proveedor para tensión y corte, pero congruente con la sobre capacidad detectada en la caracterización 
realizada a las varillas por el Organismo Nacional de Normalización y Certificación de la Construcción (ONNCCE, 2016), lo que obligaría a realizar la evaluación de las características de las varillas de acuerdo al lote y/o proveedor cuando se requiera el uso de esta capacidad superior a la nominal.

\section{Momento flexionante}

Durante el progreso de carga, inicialmente se formaron los agrietamientos primarios congruente con un mecanismo de falla por flexión; sin embargo, conforme progresó la aplicación de la carga, se evidenció una grieta típica de falla por corte que gradualmente avanzó hasta provocar el colapso.

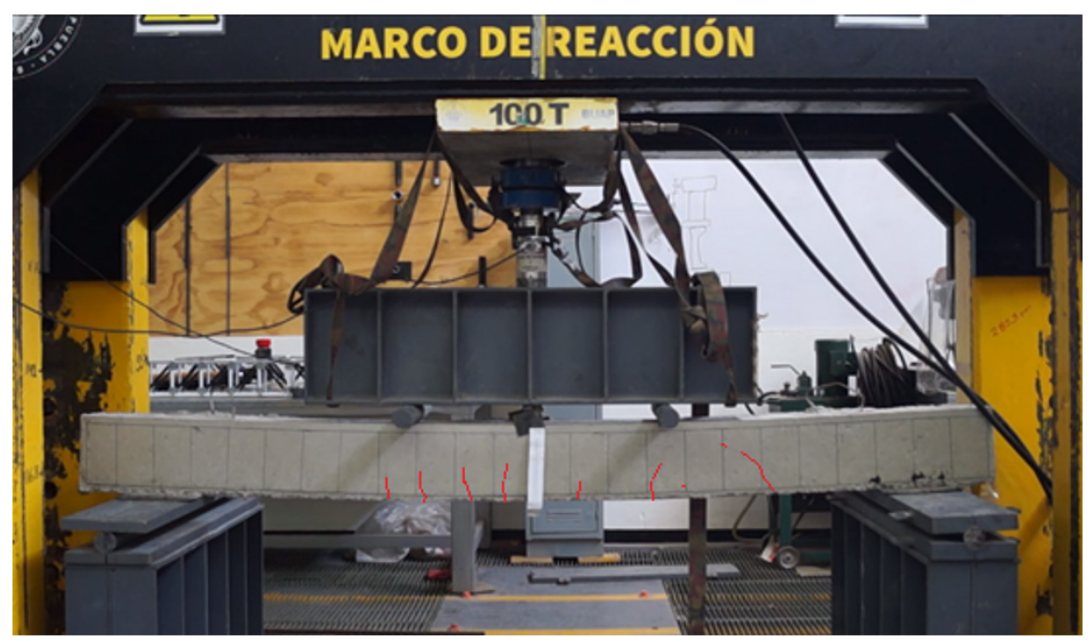

Figura 5. Progreso de Agrietamiento de viga (V4)

Un análisis comparativo de las gráficas 6a y 6b, así como de la Tabla 2 de momentos resistentes $\mathrm{Mu}$ contra deflexión $\Delta$ obtenida al centro de la viga, nos permiten observar la holgura existente entre el momento resistente (MR) calculado y el momento último a la falla $(\mathrm{Mu})$, no obstante, los factores de seguridad $\mathrm{FS}=\mathrm{Mu} / \mathrm{MR}$ no pueden ser considerados representativos porque no se alcanzó el umbral de momento resistente debido a que la falla por cortante se anticipó al colapso.

Es observable que el FS de la viga con menor refuerzo es mayor que el de mayor cuantía (Tabla 2), lo que nos hace pensar que la metodología de diseño tiende a castigar de mayor manera los refuerzos que se encuentran con menor cuantía; lo cual es congruente con la filosofía de preferir la falla del concreto a la de las varillas de refuerzo FRP enunciada por el ACI 440 1R (2015). 
Tabla 2

Factor de seguridad del diseño por flexión

\begin{tabular}{|c|c|c|c|c|c|}
\hline \multirow[b]{2}{*}{ VIGA } & \multirow[b]{2}{*}{ ARMADO } & \multicolumn{4}{|c|}{ FLEXIÓN } \\
\hline & & $\mathrm{MR}_{\mathrm{CALC}} \mathrm{Kg}-\mathrm{m}$ & MR*uKg-m & $\mathrm{MR}_{\mathrm{REAL}} \mathrm{Kg}-\mathrm{m}$ & FS \\
\hline $4(v 3)$ & $\begin{array}{c}\text { LS 2D4 } \\
\text { LI 2D4+1D6+G } \\
\text { E@15 }\end{array}$ & 302.44 & 316.55 & $1,596.67$ & 5.28 \\
\hline $5(\mathrm{v4})$ & $\begin{array}{l}\text { LS 2D6 } \\
\text { LI 3D8+G } \\
\text { E@10 }\end{array}$ & 833.91 & 938.59 & $2,246.67$ & 2.69 \\
\hline
\end{tabular}
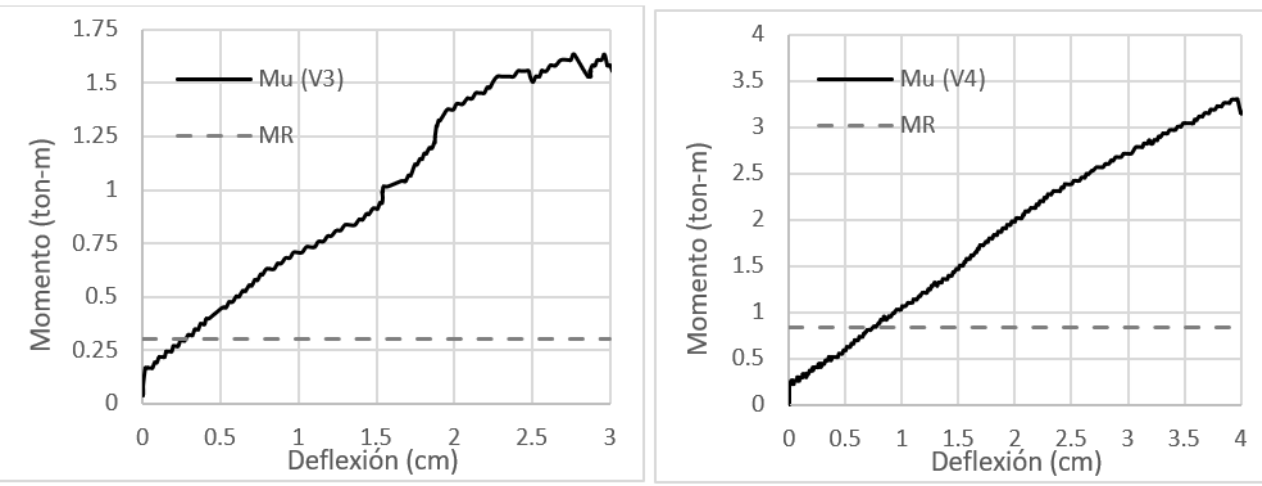

Figura 6. Curva de Momento $M x \Delta$ de las vigas: a) V3; b) V4

\section{Fuerza cortante}

En este experimento la cuantía de refuerzo propuesta fue adecuada para evaluar la falla por corte y así comparar el comportamiento del diseño mediante el enfoque tradicional referido por la Ecuación 2.2 y el planteado en la Ecuación 2 (ACI 440 1R , 2015) basado en la profundidad del eje neutro de la sección agrietada. Este experimento difiere del publicado en la Revista Pakbal No.46 (Sánchez Hernández J. A., 2019b), en el que el trabajo previo la falla por flexión rigió el colapso.

Por esta razón, en una comparación del factor de seguridad FS=Vu/VR que relaciona la resistencia alcanzada contra la evaluada podemos considerar también adecuado el diseño de la capacidad a cortante por sección agrietada, que establece el ACI 440 1R para esta acción, con los márgenes que se relacionan en la gráficas de la Figura 7 y Tabla 3, en las que se grafican junto al cortante último $(\mathrm{Vu})$ o real experimental $\left(\mathrm{VR}_{\mathrm{REAL}}\right)$, el cortante resistente evaluado mediante el mecanismo de sección agrietada propuesta por el ACI 440 y el cortante resistente de la sección bruta, usado para el acero en las NTC2017 como antes se mencionó. 
Lo anterior parece ser congruente con lo que menciona (Joaquim Luís, Díaz Pérez, \& Wainshtok Rivas, 2017) sobre la evaluación de la resistencia al corte de las vigas reforzadas con PRFV: "Las fórmulas propuestas por el ACI 440 para calcular la resistencia a cortante proporcionada por los cercos de PRFV y por el concreto fueron considerados demasiado conservadoras debido a que se basan en el dominio de compresión controlada (fallo por aplastamiento de concreto)".

\section{Tabla 3}

Factor de seguridad del diseño por corte

\begin{tabular}{ccccc}
\hline \multirow{2}{*}{ VIGA } & ARMADO & VR $_{\text {CALC }}$ Kg & $\begin{array}{c}\text { CORTANTE } \\
\text { VR }_{\text {REAL Kg }}\end{array}$ & FS \\
$\mathbf{4}(\mathbf{v 3})$ & LS 2D4 & & & \\
& LI 2D4+1D6+G & 704.88 & $2,395.00$ & 3.40 \\
& E@15 & & & \\
$\mathbf{5}(\mathbf{v 4})$ & LS 2D6 & & & \\
& LI $3 D 8+G$ & $1,230.31$ & $3,370.00$ & 2.74 \\
& E@10 & & & \\
\hline
\end{tabular}
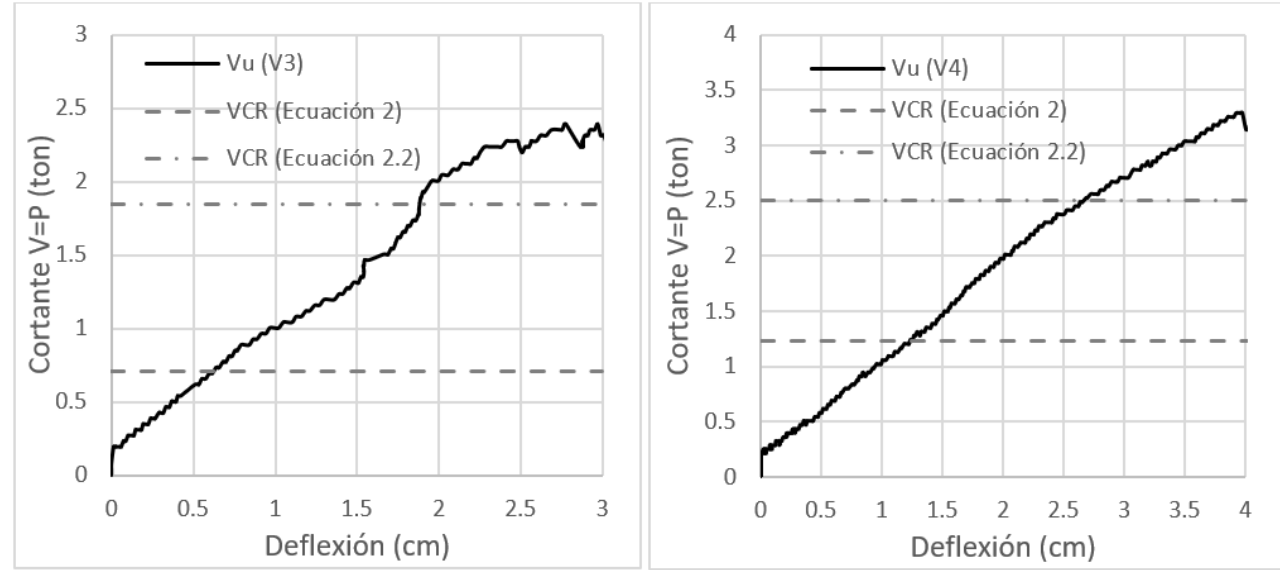

Figura 7. Curva de Cortante $V x \Delta$ de las vigas: a) V3; b) V4

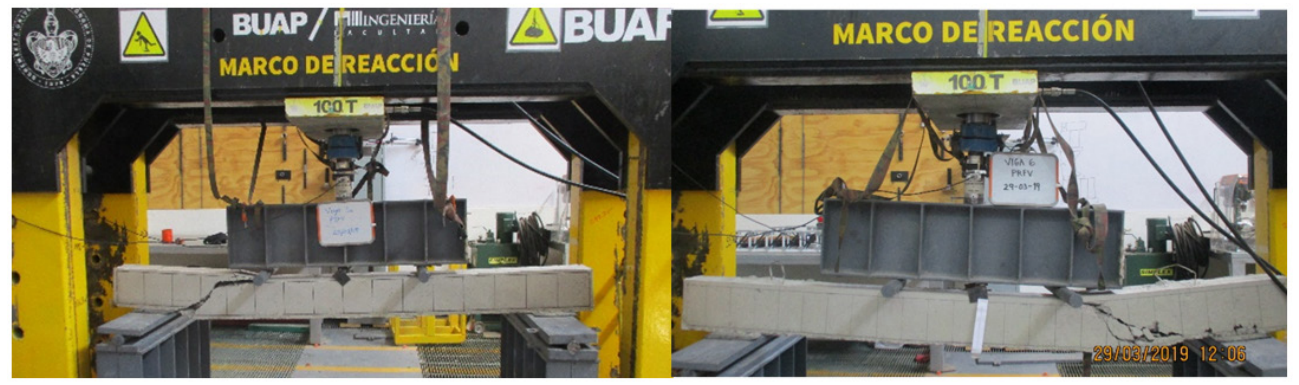

Figura 8. Falla en vigas, colapso por cortante: a) V3; b) V4 


\section{CONCLUSIONES}

1) Al comparar los resultados obtenidos en la prueba experimental con los generados conforme a los modelos analíticos validados por el ACI 440 1R (2015), basado en la filosofía de diseño con falla centrada en el concreto para este tipo de refuerzos, se puede concluir que el diseño por flexión y corte indicado en dicho código está del lado de la seguridad.

2) Es importante tomar en cuenta, que la resistencia a la tensión y corte de las barras ensayadas está por encima de las especificadas por el proveedor, valor garantizado que fue usado en las estimaciones de resistencia en estos análisis. Por lo que esta resistencia juega un papel importante en los resultados de seguridad (FS) obtenidos, y resulta trascendente en el caso de requerir del uso de esta capacidad superior en el diseño, asegurar la calidad de las varillas en el lote de PRFV a emplear.

Por tanto, aun con los factores de seguridad que aparentemente nos procura la metodología de análisis sobre los resultados obtenidos en los ensayes, no es aceptable el uso de factores de reducción y carga diferentes a los preestablecidos por las NTC (2017) y el ACI 440.1R (2017), ya que estos amortiguan el efecto de la variación de resistencia en estos materiales que puede ser muy amplio debido a su composición.

Sin duda es necesario realizar más pruebas para confirmar las deducciones aquí descritas, pero nos permite tener una mejor apreciación de las capacidades de estos materiales y justificar el auge que están tomando por sus ventajas estructurales y no estructurales.

\section{RECONOCIMIENTOS}

Los autores desean agradecer a la Benemérita Universidad Autónoma de Puebla (BUAP), a los estudiantes y prestadores, al Dr. Ing. Juan Pablo Hidalgo Toxqui catedrático de la BUAP y coordinador de los ensayes, al Ing. Luis Enrique Zepeda Morales jefe técnico de la marca POLIMEX, por permitirnos el acceso a los resultados del estudio que realiza a sus materiales. 


\section{REFERENCIAS}

ACI 440 1R . (2015). American Concrete Institute, Guide for the Design and Construction of Structural Concrete Reinforced with Fiber-Reinforced Polymer (FRP) Bars. Michigan: ACI.

GangaRao, H. V., Taly, N., \& Vijay, P. V. (2007). Reinforced Concrete Design with FRP Composites. M. Florida, U.S.: CRC Press.

Hidalgo Toxqui, J. P., Sánchez Hdez., J. A., \& et al. (2018). Evaluación del comportamiento a flexión y de los criterios de diseño de vigas de concreto subreforzadas con barras de polímeros reforzados con fibra de vidrio. Convención Científica de Ingeniería y Arquitectura. La Habana, Cuba: ISBN-978-959-261-585-4. doi:4CCI13

Joaquim Luís, F., Díaz Pérez, I. d., \& Wainshtok Rivas, H. (2017). Estructuras de hormigón armado con barras de Polímero Reforzado con Fibras de Vidrio (PRFV). Estado del arte. 11(3), 1-16.

Nanni, A., Luca, A. d., \& Jawahery, H. (2014). Reinforced Concrete with FRP Bars, Mechanics and Design. New York: CRC Press.

NTC CR. (2017). Normas Técnicas Complementarias de Concreto. Cd., de México, México.

NTC M. (2017). Normas Técnicas Complementarias para Mampostería 2017. Gaceta Oficial de Cd., de México. México.

ONNCCE. (2016). Organismo Nacional de Normalización y Certificación de la Construcción. México: Dit/401.1/15.

Sánchez Hernández, J. A. (2019). Estructuras de Concreto en Zonas Costera usando Barras de Polímero Reforzados con Fibra de Vidrio. Revista PAKBAL (45), 26-32.

Sánchez Hernández, J. A. (2019). Evaluación de los Criterios de Diseño de Vigas de Concreto Subreforzadas con Varillas de PRFV. Revista PAKBAL (46), 5-11.

Wainshtok Rivas, H., Hernández Caneiro, J. A., \& Díaz Pérez, I. d. (2015). Empleo de Barras y Telas de Mallas de PRF como refuerzo del hormigón y el ferrocemento. (978-84-17098-39-1, Ed.) Habana, Cuba: Edita.me. 